\title{
The Effects of Angular Velocity and Training Status on the Dynamic Control Equilibrium
}

\section{(a) (0) (1) $\circledast$ Version 1}

\section{Authors}

Tobias Alt ${ }^{1}$, Axel J. Knicker ${ }^{1}, 2$, Heiko Strueder ${ }^{1}$

\section{Affiliations}

1 Institute of Movement and Neuroscience, German Sport University, Cologne, Germany

2 SPRINZ Sport Performance Research Institute of New Zealand, Auckland University of Technology, New Zealand

Key words

knee joint, isokinetic dynamometry, muscle strength ratio, hamstring, quadriceps

accepted after revision November. 14. 2016

\section{Bibliography}

DOI http://dx.doi.org/10.1055/s-0042-123497

Published online: 2017 | Sports Medicine International Open

2017; 1: E23-E29

(c) Georg Thieme Verlag KG Stuttgart · New York

ISSN 2367-1890

Correspondence

Tobias Alt, M.Sc.

Institute of Movement and Neuroscience

German Sport University

Am Sportpark Müngersdorf 6

50933 Cologne

Germany

Tel.: +49/221/4982 3322, Fax: + 49/221/4973454

t.alt@dshs-koeln.de

\begin{abstract}
Thigh muscle imbalances may impair sports performance and cause injuries. Common diagnostic parameters of knee muscle balance lack practical applicability. This cross-sectional study aimed to evaluate the effects of angular velocity and training status on the dynamic control ratio at the equilibrium point representing the intersection of eccentric knee flexion and concentric knee extension moment-angle curves. 58 trained and 58 untrained male participants ( 22.1 years, $82.4 \mathrm{~kg}$ ) performed concentric and eccentric knee flexions (prone position) and extensions (supine position) on an isokinetic dynamometer operating at 30 and $150 \%$ s. Trained participants had significantly higher DCRe moments at all angular velocities compared with their untrained counterparts (trained to,150: $_{1.86}, 1.90 \mathrm{Nm} / \mathrm{kg}$; untrained $30,150: 1.56$, $1.60 \mathrm{Nm} / \mathrm{kg} ; \mathrm{p}<0.001$, partial $\eta^{2}=0.345$ ). Dynamic control equilibrium moments rose with increasing velocity $\left(p=0.001\right.$, partial $\left.\eta^{2}=0.095\right)$, whereas dynamic control equilibrium angles (trained ${ }_{30,150}: 28.9,30.8^{\circ}$; untrained ${ }_{30,150}: 26.1,27.0^{\circ}$ ) were influenced by training status $\left(p=0.004\right.$, partial $\left.\eta^{2}=0.072\right)$, but not by angular velocity $(p=0.241$, partial $\eta^{2}=0.012$ ). Dynamic control equilibrium parameters detect thigh muscle balance and reflect the trained participants' capacity to resist high eccentric knee flexor moments, especially during fast movements. Direct links to muscular loading during sprinting are conceivable, but warrant further investigation. The assessment of dynamic control equilibrium moments and angles might help physiotherapists and coaches to improve functional muscle screening, injury prevention and purposeful return to sport.
\end{abstract}

\section{Introduction}

Muscle imbalances between agonists and antagonists may impair sports performance and cause injuries such as hamstring strains $[1,14,15]$. Isokinetic dynamometry is considered to be the gold standard for the assessment of strength imbalances despite its mostly open kinetic chain, mono-articular tests and its high costs [17]. In the past 6 decades, different isokinetic evaluation techniques were proposed and implemented to assess thigh muscle balance. Conventionally, the hamstring:quadriceps moment ratio is determined from concentric (con) contractions at a given angular velocity ( $\mathrm{H}_{\mathrm{con}}$ : $\mathrm{Q}_{\mathrm{con}}$ or $\left.\mathrm{Hc:} \mathrm{Qc}\right)$ [33]. Because agonists and antagonists never contract concentrically at the same time, this parameter lacks functionality in terms of contraction mode [13]. The dynamic control ratio (DCR) - also known as the functional $\mathrm{H}: \mathrm{Q}$ ratio $[1,2]$ - remedied this problem because it represents the quotient of maximal eccentric (ecc) hamstring and maximal concentric quadriceps moment ( $\mathrm{H}_{\text {ecc }}: \mathrm{Q}_{\text {con }}$ or He:Qc) [17]. It has been frequently used in the prognosis and prevention of thigh and knee in- juries [14-16, 27]. However, its functionality and prognostic validity has been controversially discussed $[7,13,15,29]$. The major disadvantage of the DCR is that it compares moments that never act in conjunction with each other. It is well known that peak moments of eccentric flexion and concentric extension emerge at considerably different knee flexion angles of 12 and $59^{\circ}[3,4,36]$. Thus, the 'functional' $\mathrm{H}: \mathrm{Q}$ ratio (DCR) reflects a situation that never occurs during everyday movements. Due to this poor ecological validity, its application to physiological muscular loading is not reasonable [13].

Therefore, a diagnostic parameter that combines physiological contraction modes and a realistic joint angle configuration is needed to improve functional muscle screening, injury prevention and purposeful return-to-sport decisions, e. g., for hamstring strain injuries and anterior cruciate ligament tears. Coombs \& Garbutt [13] recommended to overlay the moment-angle curves of eccentric hamstring and concentric quadriceps movements and to use the intersection point of these 2 graphs as an indicator of muscular bal- 
ance around the knee joint. Although no isokinetic parameter will be able to represent functional conditions, this particular point displays the specific joint angle where the equilibrium of knee flexor and extensor moment emerges under conditions that are closer to reality than conventional and functional H:Q ratios. In the past 5 years, functional $\mathrm{H}: \mathrm{Q}$ ratios using angle-specific moments at knee flexion angles of e. g., 10, 20 and $30^{\circ}$ have received growing attention $[6,18]$. Instead of interpreting muscle balance scores at arbitrarily chosen angles with full tens values, the intersection point offers information about the distinctive joint configuration of the equilibrium of moments. Besides the moment value of this equilibrium point, the angle provides a second important measure about the muscle balance of knee flexors and extensors. Referring to the well-known dynamic control ratio (DCR), we subsequently call this point 'dynamic control ratio at the equilibrium point' or 'dynamic control equilibrium' (DCRe) for short [3]. Although this evaluation technique was introduced by Coombs \& Garbutt in 2002 and further research was announced [13], there is to our knowledge only one paper dealing with the reliability of this screening tool of thigh muscle balance [3]. DCRe moments and angles revealed the same reproducibility as traditional knee strength ratios with rising reliability at high contraction speed. At $150^{\circ} / \mathrm{s}$, high (moment: 0.906 ) to moderate (angle: 0.833 ) relative reliability scores with accordingly high absolute indices (4.9 and 6.4\%) became apparent. In spite of this quality criterion, at present there is a lack of background knowledge on the influencing factors of the DCRe moment and angle because to our knowledge, the literature does not present any evidence on this topic.

Consequently, the purpose of this study was to evaluate the effects of angular velocity and training status on DCRe parameters for the first time. We hypothesized that both DCRe moments and angles are higher in trained participants compared to their untrained counterparts. According to the results of a recently published reliability study [3], it is suggested that DCRe moments increase at higher angular velocity, whereas DCRe angles remain unchanged.

\section{Methods \\ Participants}

58 trained $(T)$ (mean \pm SD, age: $21.9 \pm 3.2$ years, height: $186.3 \pm 8.8 \mathrm{~cm}$, mass: $82.4 \pm 10.9 \mathrm{~kg}$ ) and 58 untrained (UT) (mean \pm SD, age: $22.3 \pm 4.6$ years, height: $186.2 \pm 10.5 \mathrm{~cm}$, mass: $82.3 \pm 12.7 \mathrm{~kg}$ ) healthy male participants gave their written informed consent to voluntarily join the cross-sectional study. The sample size was selected according to power calculations via $\mathrm{G}^{*}$ Power (version 3.1.9.2, Franz Kaul, Kiel, Germany). 42 regional to national level sprinters (100-400 m) as well as 12 basketball and 4 soccer players at the professional level were assigned to the trained group. They performed lower extremities' resistance training twice a week ( $\geq 2 \mathrm{~h} /$ week) containing hamstring exercises (e. g., leg curls, Nordic hamstring exercise or dead lifts) and regular sprint training for at least 5 years. The UT group consisted of sport students performing general training without focus on either muscle-specific resistance training or sprinting. The training volume of both groups was similar (8-12 h/week). All participants were free of thigh muscle and/or knee injuries within the last year. The local ethics commission verified that the require- ments of the Declaration of Helsinki were met and the authors confirm that the ethical standards were followed [23].

\section{Procedures}

To obtain reliable data, each participant performed 2 familiarisation sessions followed by a testing session which were all separated by $48-72 \mathrm{~h}$ [4]. All 3 sessions were performed in a performance laboratory and consisted of the same procedure. After determining their mass, the participants performed an individual warm-up (jogging, dynamic stretching) for $10 \mathrm{~min}$. This general warm-up was succeeded by the participant's positioning on the active isokinetic dynamometer (IsoMed 2000, D\&R Ferstl GmbH, Hemau, Germany). A double shin pad for unilateral knee movements was attached to the motor-driven axis. In a pre-activated muscular state at $90^{\circ}$ knee flexion, the dynamometer axis was aligned with the participants' lateral femoral epicondyle by aid of a laser pointer [5]. The distal part of the pad was fixed by straps approximately $2-3 \mathrm{~cm}$ proximal to the medial malleolus. After a gravity correction measurement, the participants performed an isokinetic warm-up consisting of 15 submaximal concentric and eccentric repetitions of the respective muscle group. The participants took off their shoes to minimize accelerative inaccuracies.

The testing order of the 4 conditions (left and right flexion and extension) was stratified such that limbs were alternately tested and slow velocities were executed prior to fast ones [37]. For each condition, both concentric and eccentric movements were conducted at 2 different angular velocities (30 and $150^{\circ} / \mathrm{s}$ ). The reliability of this protocol and the derived parameters has been previously assessed as high [3,4]. To attain the largest possible isokinetic range of motion, a very quick acceleration and a hard deceleration were chosen. Each set consisted of 5 repetitions (2 practice, 3 with maximum effort) performed as discrete movements in a single direction [32]. The return of the involved leg into the starting position always occurred passively at $120^{\circ} \mathrm{s}$. The inter-set rest interval was set at $1 \mathrm{~min}$ [8]. Strong verbal encouragement promoted maximum effort.

For knee flexion, the participants lay prone $\left(0^{\circ}\right.$ hip flexion $)$ and pushed their trunk with their hands gripping the lounger handles ( $\triangleright$ Fig. 1). The knee flexion's range of motion was set at $110^{\circ}$ starting at full knee extension $\left(0^{\circ}\right)$. Knee extensions were performed in the supine position ( $0^{\circ}$ hip flexion) from $90^{\circ}$ to $0^{\circ}$ knee flexion. The prone and supine positioning of the participants was chosen to simulate muscle fibre lengths that occur during the mid-stance phase of running and sprinting $[6,20,21]$. Hand grips beside the hip provided sufficient stability $[5,22]$. In order to examine the largest range of the respective condition, the degree of movement differed between knee flexion and extension [3,4] ( Fig. 1).

Raw data were recorded by the manufacturer's software at a sampling rate of $200 \mathrm{~Hz}$ (IsoMed analyse V.2.0, D\&R Ferstl GmbH, Hemau, Germany). Data were stored as ASCII files which were processed by a self-developed software $(\mathrm{C}++)$ isolating the isokinetic range of motion ( $\pm 1 \%$ ). To reduce oscillations of the derived moment-angle curves, a $5^{\text {th }}$ order Butterworth low-pass filter with a cut-off frequency of $6 \mathrm{~Hz}$ was applied. Relevant test parameters were computed from the obtained moment-angle curves of the 3 maximum repetitions: highest gravity-corrected peak moments (PM), angles of peak moment (APM) as well as DCRe moments and 


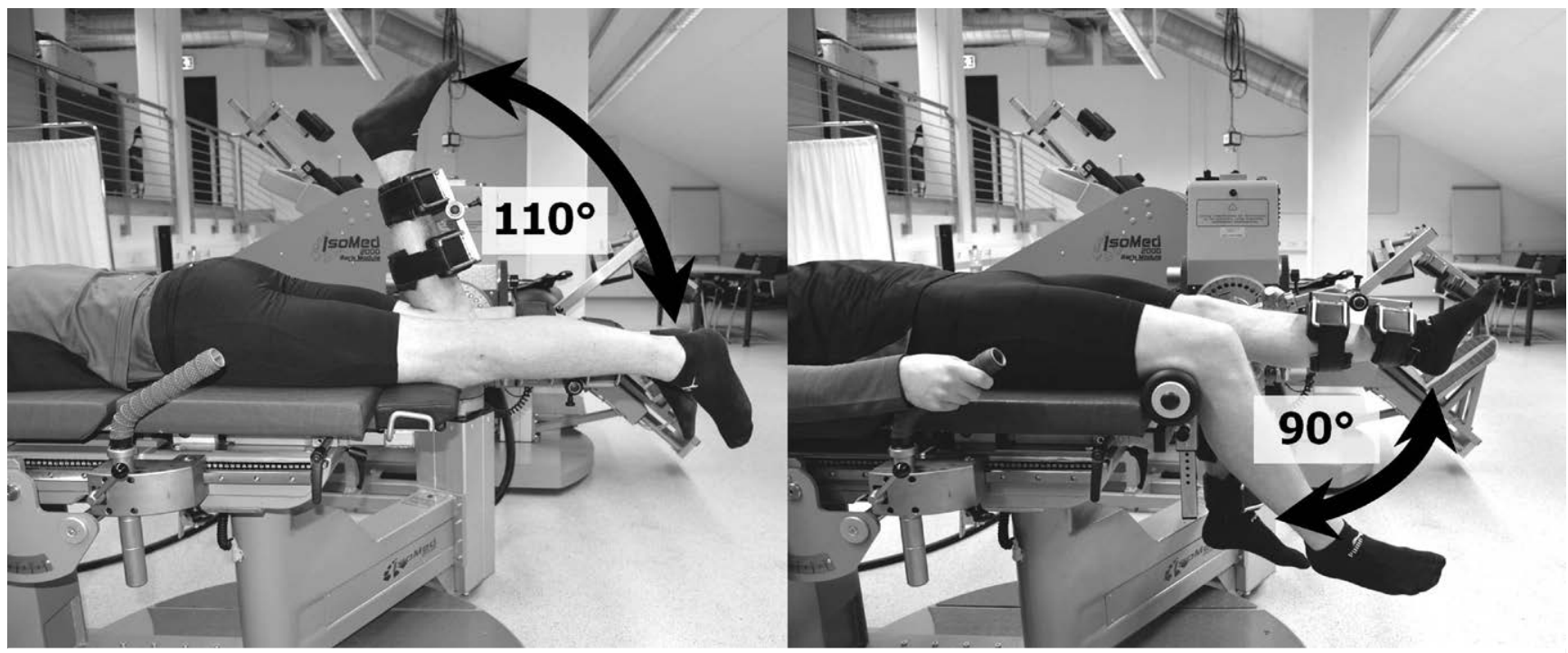

Fig. 1 Knee flexions (left) and extensions (right) were conducted in prone and supine position across the largest possible range of motion. Hand grips provided sufficient stabilisation to the lounger.

angles. Normalization to body mass enabled inter-individual comparison [26].

\section{Statistical analyses}

Normal distribution and variance homogeneity were confirmed by the Kolmogorov-Smirnov $(\alpha \leq 0.05)$ and Levene's test $(\alpha \leq 0.10)$. As bilateral asymmetries were beyond the scope of the study, the results of both limbs were averaged for each participant. ANOVAs with repeated measures served to detect significant effects of training status, muscle, contraction mode and angular velocity on the PM and APM as well as the influence of angular velocity and training status on DCRe moments, angles and on DCR. Bonferroni post hoc tests $(\alpha \leq 0.05)$ determined the actual $p$-values of the examined factors. Effect sizes are presented as partial eta-squared (partial $\left.\eta^{2}\right)$ and indicate the meaningfulness of the effects. According to Cohen [12], a partial $\eta^{2}$ of $0.02,0.13$ and 0.26 represents a small, medium and large effect size. All statistical tests were conducted with SPSS V22.0 (SPSS Inc., Chicago, Illinois, USA).

\section{Results}

$\checkmark$ Fig. 2 demonstrates the moment ( $\vee$ Fig. 2a) and functional H:Q ratio-knee flexion angle curves ( $\vee$ Fig. $\mathbf{2 b}$ ) of the trained group for both angular velocities, illustrating the distinctive nature of the DCRe where the eccentrically active hamstrings would be able to fully brake the extension moment produced by the concentrically contracting quadriceps [13].

The typical moment-angular velocity relations ( $\vee$ Fig. 3a) showed that trained participants generated consistently higher PMs during maximal concentric and eccentric knee movements at 30 and $150^{\circ} / \mathrm{s}$ compared to their untrained counterparts for both knee flexors and extensors $\left(T>U T ; p<0.001\right.$, partial $\eta^{2}=0.227$ ) ( $\vee$ Fig. 3a). APMs were independent of training status $(p=0.697$, partial $\eta^{2}=0.003$ ), but significantly differed with regard to muscle group (flex<ext; $p<0.001$, partial $\eta^{2}=0.988$ ) and to the interac- tion of training status and muscle group $(p<0.003$, partial $\left.\eta^{2}=0.080\right)(\triangleright$ Fig. 3b).

- Fig. 2a illustrates the moment-angle relations obtained from concentric knee extensions and eccentric flexions at 30 and $150 \%$ s. The DCR of angle-specific moments considerably changed throughout the range of motion ( $\triangleright$ Fig. $\mathbf{2 b}$ ). However, its shape did not significantly diverge between different angular velocities. DCRe moments were significantly higher for trained participants at all angular velocities $\left(p<0.001\right.$, partial $\left.\eta^{2}=0.345\right)\left(T_{30,150}: 1.86,1.90 \mathrm{Nm} /\right.$ $\left.\mathrm{kg} ; \mathrm{UT}_{30,150}: 1.56,1.60 \mathrm{Nm} / \mathrm{kg}\right)$. DCRe moments were higher at $150^{\circ} / \mathrm{s}$ compared to $30^{\circ} / \mathrm{s}\left(p=0.001\right.$, partial $\left.\eta^{2}=0.095\right)$. In contrast, DCRe angles were affected by training status $(p=0.004$, partial $\left.\eta^{2}=0.072\right)$, but not by angular velocity $\left(p=0.241\right.$, partial $\left.\eta^{2}=0.012\right)$ $\left(T_{30,150}: 28.9,30.8^{\circ} ; U_{30,150}: 26.1,27.0^{\circ}\right)$. However, the effect of training status on DCRe angles emerged only at $150^{\circ} / \mathrm{s}$ where the trained group had significantly greater values compared to the untrained group. For both DCRe parameters, no significant interaction of training status and velocity became apparent ( $\vee$ Fig. 4a). DCR was unaffected by training status $\left(T_{30,150}: 0.60\right.$, $0.74 \mathrm{Nm} / \mathrm{kg} ; \mathrm{UT}_{30,150}: 0.58,0.71 \mathrm{Nm} / \mathrm{kg} ; \mathrm{p}=0.081$, partial $\left.\eta^{2}=0.011\right)$, but significantly increased with angular velocity $\left(p<0.001\right.$, partial $\left.\eta^{2}=0.609\right)$.

\section{Discussion \\ Physiological rationale for the dynamic control equilibrium (DCRe)}

The present cross-sectional study aimed to improve the understanding of the DCRe representing the intersection of eccentric knee flexion and concentric knee extension moment-angle curves. This yet relatively unknown screening tool comprises the physiological properties of the moment-angle relationships ( $\vee$ Fig. 2 a), PM- ( Fig. 3a) and APM-angular velocity ( Fig. 3b) $[13,36]$. In addition to the effect of muscle group, angular velocity and contraction mode, athletes' training status obviously lead to a signifi- 

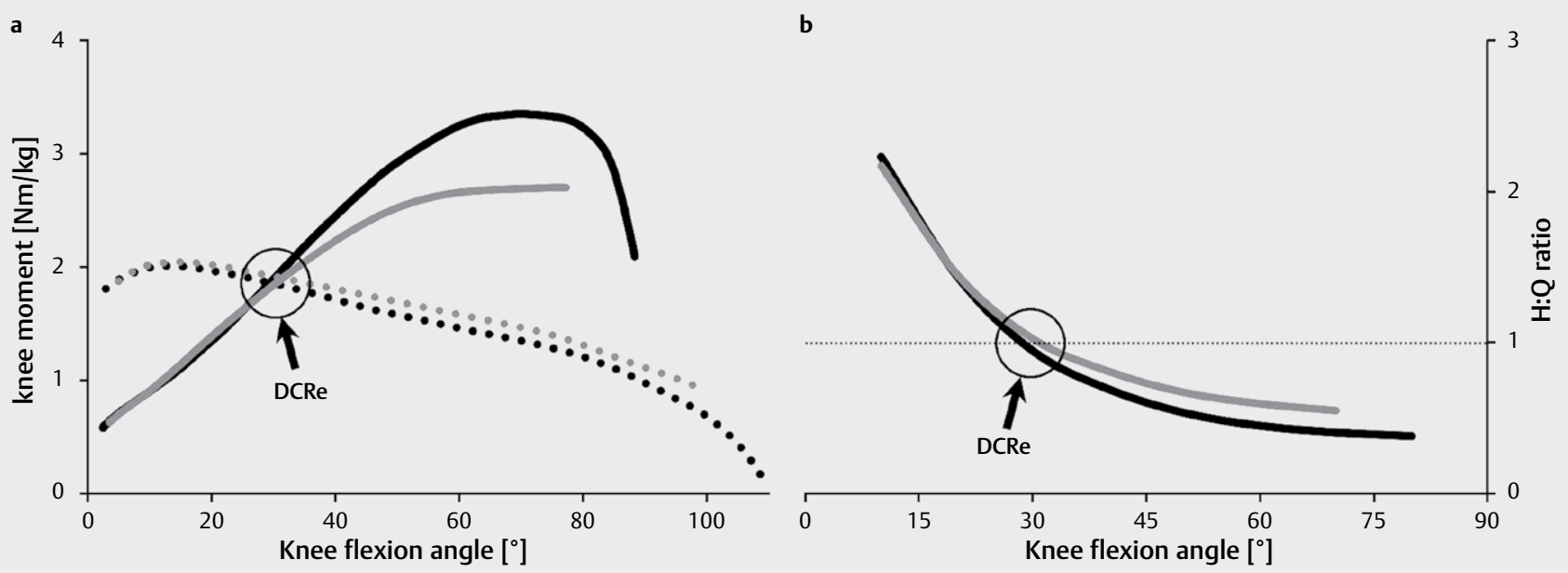

- Fig. 2 a Mean moment-angle relationships of concentric knee extension (solid lines) and eccentric flexion (dashed lines) and $\mathbf{b}$ functional H:Q ratios at 30 and $150^{\circ} / \mathrm{s}$ (black to grey) of trained participants reaching their DCRe at approximately 29 and $31^{\circ}$.
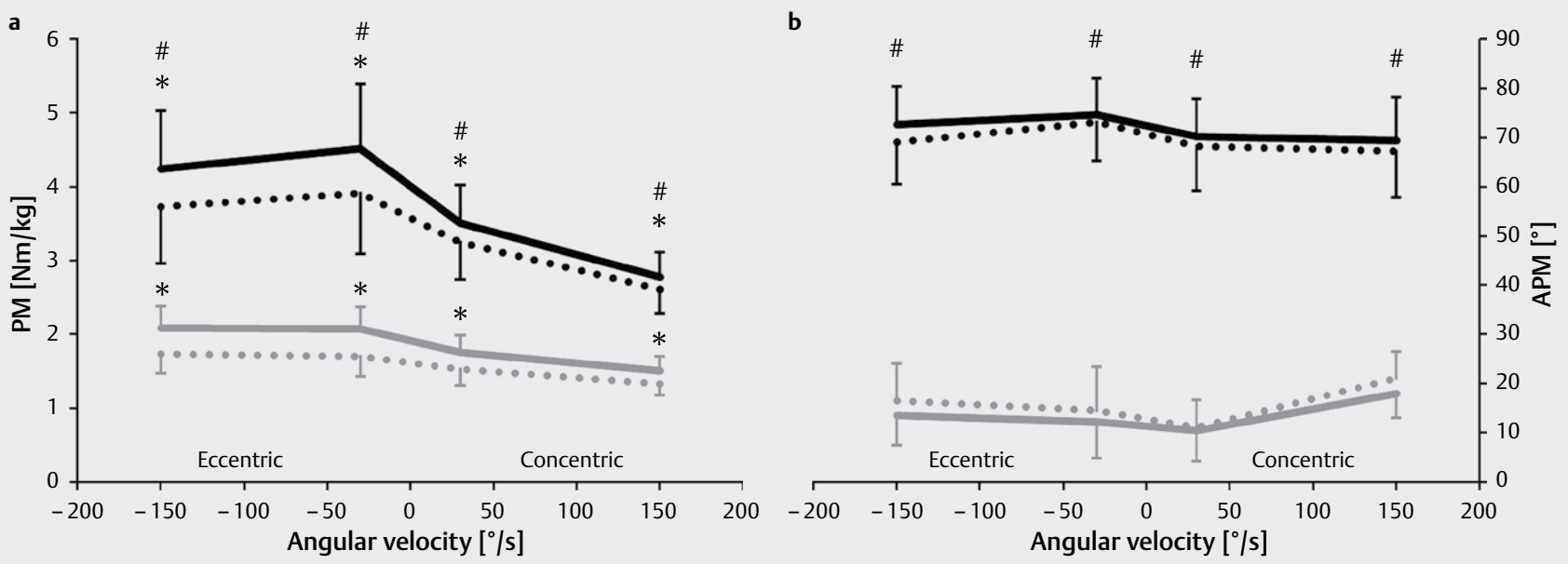
Fig. 3 Angular velocity relationships expressed as means and standard deviations of a peak moments (PM) and $\mathbf{b}$ angles of peak moment (APM)
obtained from concentric and eccentric knee extensions (black) and flexions (grey) of trained (solid lines) and untrained (dashed lines) participants at
30 and $150^{\circ} / \mathrm{s} .{ }^{*}$ and ${ }^{\#}$ indicate significant effects $(\mathrm{p} \leq 0.05)$ of training status and muscle group, respectively.

cantly higher PM throughout all concentric and eccentric contraction velocities ( $\mathbf{F i g}$. 3a). However, APM remained unaffected by the specific training regimen itself and considerably differed between knee flexors (range $=10.5-21.1^{\circ}$ ) and extensors (range $\left.=67.2-74.6^{\circ}\right)$ ( $\triangleright$ Fig. 3b). This physiological discrepancy of APM is well-known $[3,4,36]$ and queries the calculation and meaningful interpretation of 'functional' $\mathrm{H}$ :Q ratios. To avoid the comparison of moments that are generated at different joint angles $[14,36]$, Coombs \& Garbutt [13] introduced the DCRe as a diagnostic parameter for more functional analyses of reciprocal thigh muscle performance ( $\mathbf{F i g}$. 2 a, b). Since functional $\mathrm{H}: \mathrm{Q}$ ratios using angle-specific moments incorporate the advantage of angle conformity, they received growing attention $[6,18]$. However, instead of interpreting muscle balance scores at arbitrarily chosen angles with full tens values (e. g., 10, 20 and $30^{\circ}$ ), the DCRe provides information about the distinctive joint configuration of the equilibrium of moments. In the authors' opinion, it is more important to know the range of motion in which the eccentrically acting hamstrings are able to withstand the concentric quadriceps' action rather than to know the values of functional $\mathrm{H}$ :Q ratios close to knee extension ( $\vee$ Fig. $\mathbf{2 a} \mathbf{b}$ b).

In spite of its better ecological validity in terms of contraction mode and joint angle configuration compared with conventional and functional $\mathrm{H}: \mathrm{Q}$ ratios, DCRe moments and angles have not been previously analysed although both DCRe parameters possessed similar reproducibility $[3,6,25]$.

\section{Effect of angular velocity and training status on DCRe moments and angles}

As hypothesized, DCRe moments were significantly higher at greater movement velocity and for trained participants at all angular velocities. In contrast to the DCR, DCRe moments detected the 


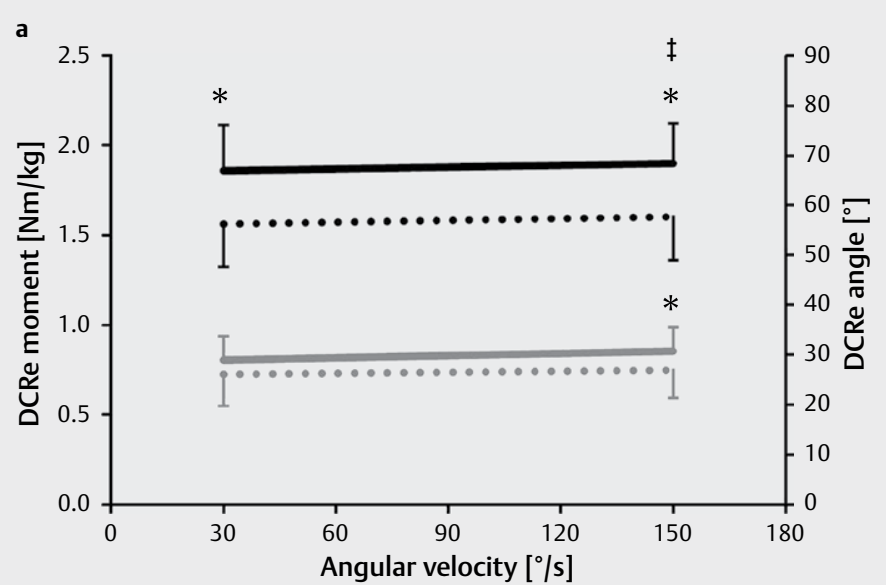

b

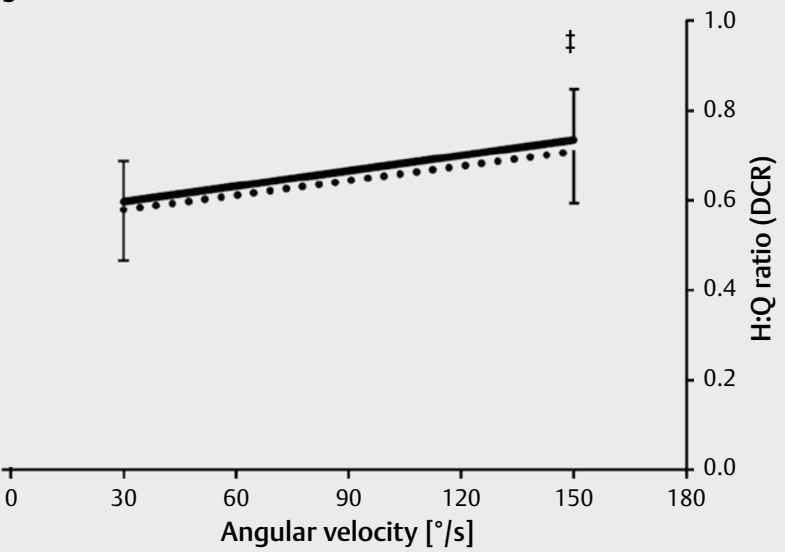

- Fig. 4 Mean values and standard deviations of a DCRe moments (black) and angles (grey) as well as b DCR values of trained (solid lines) and untrained (dashed lines) participants at 30 and $150^{\circ} / \mathrm{s}$. ${ }^{*}$ and $¥$ indicate significant effects $(p \leq 0.05)$ of training status and angular velocity, respectively.

increased capacity of trained participants to produce high eccentric knee flexor moments, especially during fast knee extensions ( $\triangleright$ Fig. 4a,b). Consequently, DCRe does not just rate thigh muscle balance on a yes-no basis but provides quantitative data differentiating the training status of analysed athletes or patients.

DCRe angles were not affected by angular velocity but by training status. This finding confirmed the assumption of Coombs \& Garbutt [13] that DCRe should be shifted towards a more flexed knee joint position by training. With regard to the present results, the adaptation to specific resistance and sprint training was a rise of DCRe moments with concomitantly greater DCRe angles. The latter was especially true at high angular velocity ( $\triangleright \mathbf{F i g}$. 4a) and emphasizes the increased ability of trained participants to conduct fast and forceful knee extensions without overloading and potentially injuring their hamstrings ( $\triangleright$ Fig. 2a, b). Thus, the present results emphasize the functional value of both DCRe parameters and provide a first collection of target values which might help physiotherapists and coaches to improve functional screening of thigh muscle balance.

\section{Link of DCRe parameters to muscular loading in sprinting}

To assess the practicability of DCRe parameters, the present results were compared post hoc with data obtained from biomechanical sprint analyses. Thereby, we focus on the late swing phase of the running cycle when the hamstrings undergo an active lengthening and absorb kinetic energy throughout fast and forceful knee extensions [11,24]. The kinematic and kinetic results of previously published motion analyses show 3 striking similarities between DCRe parameters and muscular loading during sprinting. First, the amount of DCRe moments generated by trained participants at $150^{\circ} / \mathrm{s}$ ( Fig. 4 ) is similar to the peak eccentric knee flexor moments during the late swing phase of sprinting, reaching 1.8$2.5 \mathrm{Nm} / \mathrm{kg}[28,35]$. Second, DCRe angles correspond to the knee joint angle where peak eccentric flexor moments were detected via inverse dynamics occurring at $\sim 25-30^{\circ}$ of knee flexion ( $\triangleright$ Fig. 4) $[10,34]$. Third, the negative work and peak moments imposed on the hamstrings' muscle-tendon unit prior to foot-strike increase with running speed [10]. This feature reveals that faster athletes might have an increased capacity to resist high eccentric flexor moments during fast knee extensions expressed by significantly higher DCRe moments ( $\triangleright$ Fig. 4) [35]. This is the result of greater specific strength capacities as well as superior coordination and activation patterns of trained athletes [21, 30, 31, 35]. In summary, the abovementioned similarities between DCRe parameters and sprint-related muscular loading suggest a high practical relevance of this screening tool [3].

\section{Limitations}

Even if DCRe parameters are superior to the conventional and functional H:Q ratios in terms of contraction mode, joint angle configuration and detection of training status, this evaluation method suffers from methodological limitations impeding a realistic reflection of the actual muscular loading during e. g., sprinting. The analysed angular velocities were much lower compared with the late swing phase of maximum velocity sprinting $\left(900-1200^{\circ} / \mathrm{s}\right)[28,35]$. However, such high velocities cannot be achieved by isokinetic dynamometers. Even if viable, the isokinetic range of motion would be too small to get results of the particular knee angles $\left(\sim 25-30^{\circ}\right)$. Furthermore, both mechanical (e. g., valid velocity adjustment) and psycho-physiological reasons (e. g., injury scare) would impair the validity of such data [9]. The isokinetic tests were conducted with extended hip joints. However, peak flexor moments during sprinting usually emerge at $\sim 60-70^{\circ}$ hip flexion [34]. This problem might be solved by pads predetermining a certain amount of hip flexion. Even if the calculation of DCRe parameters is more difficult compared to conventional and functional $\mathrm{H}: \mathrm{Q}$ ratios, their common and broadly used application and further investigation is recommended [3].

\section{Perspectives}

Further research is needed to verify the 3 suggested links between DCRe parameters and sprint kinematics and kinetics. It was beyond the scope of this study to detect susceptible athletes for hamstring strains. However, it is desired to analyse the muscular capacities of 
larger cohorts including pre- and post-injured participants. Prospective studies should investigate the predictive value of DCRe in the valid assessment of muscular imbalances predisposing muscle and knee injuries $[19,21,30]$. This would provide additional information about the practicability and suitability of DCRe in the functional and meaningful assessment of thigh muscle balance concerning the prevention and prognosis of related injuries as well as purposeful return to sport [14].

\section{Conclusions}

A functional and reliable diagnostic parameter to assess potential muscular imbalances between knee flexors and extensors might contribute to better sports performance and reduced injury susceptibility. The physiological rationale of commonly applied screening tools such as the conventional and functional $\mathrm{H}$ :Q ratio has been controversially discussed with regard to contraction mode and joint configuration. This study aimed to improve the understanding of the dynamic control ratio at the equilibrium point (DCRe) representing the intersection of eccentric knee flexion and concentric knee extension moment-angle curves during maximum isokinetic exercise. Higher DCRe moments and angles displayed the increased capacity of trained participants to resist high eccentric knee flexor moments, especially during fast knee extensions. With regard to this study, the DCRe is a functional and reliable evaluation technique to detect potential thigh muscle imbalances by providing quantitative data distinguishing the training status of analysed athletes or patients. Accordances between DCRe parameters and kinematic and kinetic sprint data suggest direct links to muscular loading during sprinting. The clinical relevance of this diagnostic parameter concerning injury prevention and prognosis of hamstring strain injuries warrants further investigation. In spite of this, we recommend the assessment of DCRe parameters within isokinetic strength tests for functional and reliable diagnosis of potential thigh muscle imbalances.

\section{Acknowledgements}

The authors would like to thank all participants who volunteered to participate in this study and demonstrated great motivation and commitment.

\section{Conflict of interest}

There is no conflict of interest.

\section{References}

[1] Aagaard P, Simonsen EB, Magnusson SP, Larsson B, Dyhre-Poulsen P. A new concept for isokinetic hamstring: quadriceps muscle strength ratio. Am J Sports Med 1998; 26: 231-237

[2] Aagaard P, Simonsen EB, Trolle M, Bangsbo J, Klausen K. Isokinetic hamstring/quadriceps strength ratio: influence from joint angular velocity, gravity correction and contraction mode. Acta Physiol Scand 1995; 154: 421-427
[3] Alt T, Knicker AJ, Struder HK. The dynamic control ratio at the equilibrium point (DCRe): introducing relative and absolute reliability scores. J Sports Sci 2016; 23: 1-6 [Epub ahead of print]

[4] Alt T, Knicker AJ, Strüder HK. Factors influencing the reproducibility of isokinetic knee flexion and extension test findings. Isokinet Exerc Sci 2014; 22: 333-342

[5] Alt T, Richert M, Schwarz YM, Knicker AJ, Strüder HK. Minimal fixation suffices for supine isokinetic knee extension: a kinetic and 3D kinematic analysis. Med Sci Sports Exerc 2016;48: 887

[6] Ayala F, De Ste Croix M, Sainz de Baranda P, Santonja F. Absolute reliability of hamstring to quadriceps strength imbalance ratios calculated using peak torque, joint angle-specific torque and joint ROM-specific torque values. Int J Sports Med 2012; 33: 909-916

[7] Bennell K, Wajswelner H, Lew P, Schall-Riaucour A, Leslie S, Plant D, Cirone J. Isokinetic strength testing does not predict hamstring injury in Australian Rules footballers. Br J Sports Med 1998; 32: 309-314

[8] Blazquez IN, Warren BL, O’Hanlon AM, Silvestri LR. An adequate interset rest period for strength recovery during a common isokinetic test. J Strength Cond Res 2013; 27: 1981-1987

[9] Caruso JF, Brown LE, Tufano JJ. The reproducibility of isokinetic dynamometry data. Isokinet Exerc Sci 2012; 20: 239-253

[10] Chumanov ES, Heiderscheit BC, Thelen DG. The effect of speed and influence of individual muscles on hamstring mechanics during the swing phase of sprinting. J Biomech 2007; 40: 3555-3562

[11] Chumanov ES, Schache AG, Heiderscheit BC, Thelen DG. Hamstrings are most susceptible to injury during the late swing phase of sprinting. $\mathrm{Br}$ J Sports Med 2012; 46: 90

[12] Cohen J. Statistical power analysis for the behavioral sciences. $2^{\text {nd }}$ ed. Hillsdale, NJ: Erlbaum; 1988

[13] Coombs R, Garbutt G. Developments in the use of the hamstring/ quadriceps ratio for the assessment of muscle balance. J Sports Sci Med 2002; 1: 56-62

[14] Dallinga JM, Benjaminse A, Lemmink KA. Which screening tools can predict injury to the lower extremities in team sports? A systematic review. Sports Med 2012; 42: 791-815

[15] De Ste Croix M, Deighan M, Armstrong N. Assessment and interpretation of isokinetic muscle strength during growth and maturation. Sports Med 2003; 33: 727-743

[16] De Ste Croix M, Deighan M, Armstrong N. Functional eccentric-concentric ratio of knee extensors and flexors in pre-pubertal children, teenagers and adult males and females. Int J Sports Med 2007; 28 : 768-772

[17] Dvir Z, Eger G, Halperin N, Shklar A. Thigh muscle activity and anterior cruciate ligament insufficiency. Clin Biomech (Bristol, Avon) 1989; 4 : 87-91

[18] El-Ashker S, Carson BP, Ayala F, De Ste Croix M. Sex-related differences in joint-angle-specific functional hamstring-to-quadriceps strength ratios. Knee Surg Sports Traumatol Arthrosc 2015; ]ul 7 [Epub ahead of print]

[19] Goldman EF, Jones DE. Interventions for preventing hamstring injuries: a systematic review. Physiotherapy 2011; 97: 91-99

[20] Guex K, Gojanovic B, Millet GP. Influence of hip-flexion angle on hamstrings isokinetic activity in sprinters. J Athl Train 2012; 47: 390-395

[21] Guex K, Millet GP. Conceptual framework for strengthening exercises to prevent hamstring strains. Sports Med 2013; 43: 1207-1215

[22] Hanten WP, Ramberg CL. Effect of stabilization on maximal isokinetic torque of the quadriceps femoris muscle during concentric and eccentric contractions. Phys Ther 1988; 68: 219-222

[23] Harriss DJ, Atkinson G. Ethical standards in sport and exercise science research: 2016 update. Int J Sports Med 2015; 36: 1121-1124 
[24] Heiderscheit BC, Sherry MA, Silder A, Chumanov ES, Thelen DG. Hamstring strain injuries: recommendations for diagnosis, rehabilitation, and injury prevention. J Orthop Sports Phys Ther 2010; 40: 67-81

[25] Impellizzeri FM, Bizzini M, Rampinini E, Cereda F, Maffiuletti NA. Reliability of isokinetic strength imbalance ratios measured using the Cybex NORM dynamometer. Clin Physiol Funct Imaging 2008; 28: 113-119

[26] Jaric S. Role of body size in the relation between muscle strength and movement performance. Exerc Sport Sci Rev 2003; 31: 8-12

[27] Jenkins ND, Hawkey M], Costa PB, Fiddler RE, Thompson B], Ryan ED, Smith D, Sobolewski EJ, Conchola EC, Akehi K, Cramer JT. Functional hamstrings: quadriceps ratios in elite women's soccer players. J Sports Sci 2013; 31: 612-617

[28] Johnson MD, Buckley JG. Muscle power patterns in the mid-acceleration phase of sprinting. J Sports Sci 2001; 19: 263-272

[29] Kellis E, Baltzopoulos V. Isokinetic eccentric exercise. Sports Med 1995; 19: 202-222

[30] Malliaropoulos N, Mendiguchia J, Pehlivanidis H, Papadopoulou S, Valle $X$, Malliaras P, Maffulli N. Hamstring exercises for track and field athletes: injury and exercise biomechanics, and possible implications for exercise selection and primary prevention. Br J Sports Med 2012; 46: 846-851
[31] Mendiguchia J, Alentorn-Geli E, Brughelli M. Hamstring strain injuries: are we heading in the right direction? $\mathrm{Br}$ J Sports Med 2012; 46: $81-85$

[32] Rothstein JM, Lamb RL, Mayhew TP. Clinical uses of isokinetic measurements. Critical issues. Phys Ther 1987; 67: 1840-1844

[33] Steindler A. Kinesiology of the Human Body under Normal and Pathological Conditions. Springfield, IL: Charles C. Thomas Publisher. 1955

[34] Thelen DG, Chumanov ES, Best TM, Swanson SC, Heiderscheit BC. Simulation of biceps femoris musculotendon mechanics during the swing phase of sprinting. Med Sci Sports Exerc 2005; 37: 1931-1938

[35] Vardaxis V, Hoshizaki TB. Power patterns of the leg during the recovery phase of the sprinting stride for advanced and intermediate sprinters. Int J Sport Biomech 1989; 5: 332-339

[36] Westing SH, Seger JY. Eccentric and concentric torque-velocity characteristics, torque output comparisons, and gravity effect torque corrections for the quadriceps and hamstring muscles in females. Int J Sports Med 1989; 10: 175-180

[37] Wilhite MR, Cohen ER, Wilhite SC. Reliability of concentric and eccentric measurements of quadriceps performance using the KIN-COM dynamometer: the effect of testing order for three different speeds. J Orthop Sports Phys Ther 1992; 15: 175-182 